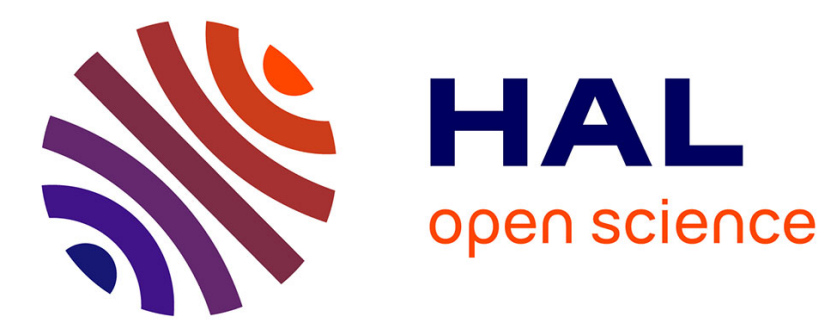

\title{
Bounded Rayleigh Mixture Model for Ultrasound Image Segmentation
}

\author{
Hui Bi, Hui Tang, Hua Zhong Shu, Jean-Louis Dillenseger
}

\section{To cite this version:}

Hui Bi, Hui Tang, Hua Zhong Shu, Jean-Louis Dillenseger. Bounded Rayleigh Mixture Model for Ultrasound Image Segmentation. 8th International Conference on Graphic and Image Processing, Oct 2016, Tokyo, Japan. 10.1117/12.2266963 . inserm-01426910

\section{HAL Id: inserm-01426910 https://www.hal.inserm.fr/inserm-01426910}

Submitted on 5 Jan 2017

HAL is a multi-disciplinary open access archive for the deposit and dissemination of scientific research documents, whether they are published or not. The documents may come from teaching and research institutions in France or abroad, or from public or private research centers.
L'archive ouverte pluridisciplinaire HAL, est destinée au dépôt et à la diffusion de documents scientifiques de niveau recherche, publiés ou non, émanant des établissements d'enseignement et de recherche français ou étrangers, des laboratoires publics ou privés. 


\title{
Bounded Rayleigh Mixture Model for Ultrasound Image Segmentation
}

\author{
H. Bi ${ }^{1}$, H. Tang ${ }^{1}$, H. Z. Shu ${ }^{1,2, *}$, and J. L. Dillenseger ${ }^{2,3}$ \\ ${ }^{1}$ School of Computer Science and Engineering, Southeast University, Nanjing, China \\ ${ }^{2}$ Centre de Recherche en Information Biomédicale Sino-Français (CRIBs), 35000 Rennes, France \\ ${ }^{3}$ Université de Rennes I, 35000 Rennes, France \\ Email: shu.list@seu.edu.cn
}

\begin{abstract}
The finite mixture model based on the Gaussian distribution is a flexible and powerful tool to address image segmentation. However, in the case of ultrasound images, the intensity distributions are non-symmetric whereas the Gaussian distribution is symmetric. In this study, a new finite bounded Rayleigh distribution is proposed. One advantage of the proposed model is that Rayleigh distribution is non-symmetric which has ability to fit the shape of medical ultrasound data. Another advantage is that each component of the proposed model is suitable for the ultrasound image segmentation. We also apply the bounded Rayleigh mixture model in order to improve the accuracy and to reduce the computational time. Experiments show that the proposed model outperforms the state-of-art methods on time consumption and accuracy.
\end{abstract}

Keywords: Rayleigh mixture model, ultrasound image segmentation, bounded mixture model

\section{INTRODUCTION}

Image segmentation is the key issue in computer version and machine learning. Many methods have been proposed for image segmentation. These methods divide into four categories: edge detection, threshold, clustering and region growing [1]. Clustering is a useful tool for image segmentation, which assigns the same label to pixels having the same characteristic. The outstanding method of clustering is finite mixture model (FMM). Image segmentation and classification based on FMM have been reported [2].

Gaussian Mixture Model (GMM) is the most commonly used case of FMM by assuming the intensity distributions follows Gaussian distributions. The main advantage of GMM is that it is easy to implement and it has only a few parameters to be estimated [3]. GMM has been applied in various applications, such as audio processing, video applications, and image segmentation. However, the case of ultrasound (US) image segmentation is more particular because of speckle pattern [4]. The intensity distributions of US images are non-symmetric whereas Gaussian distribution is symmetric. Several non-symmetric statistical distributions, including Rician [5], K- [6] or Nakagami [7], have been used to model the distribution of scatterers in US. Some authors proposed to adapt the FMM segmentation framework for US analysis by estimating a Rayleigh mixture model (RMM) [8]. It was shown that the Rayleigh distribution is more suitable to model the intensity distribution of US images. Moreover, the RMM has only one parameter to be estimated.

In order to better fit different shapes of data, many models are introduced. Nguyen et al. exploited the bounded Student's $t$ Mixture Model (BSMM) [9] for Synthetic Aperture Radar (SAR) images segmentation since Student's $t$ distribution is suitable for SAR images. Since the case of ultrasound (US) image segmentation is more particular, Rayleigh distribution is more suitable to apply. Motivated by the previous research work, we propose a new finite bounded Rayleigh Mixture Model (BRMM) scheme for ultrasound image segmentation. In the proposed BRMM, each image component is viewed as a mixture of Rayleigh distribution. The expected advantages of the proposed method are: (1) Rayleigh distributions are well adapted to US images; (2) each component of the proposed model can be used to demonstrate the whole image; (3) segmentation accuracy is improved and the time consumption of our model is attractive for clinical application.

The reminder of this paper is organized as follows. Section 2 presents the proposed model and elaborates the parameters' estimation. Section 3 provides the experimental results, and concluding remarks are given in Section 4. 


\section{PROPOSED METHOD}

The general FMM is briefly reviewed at first in this section. Under the independence assumption, RMM is defined as:

$$
f\left(x_{i} \mid \Theta\right)=\sum_{j=1}^{L} \pi_{j} p\left(x_{i} \mid \tau_{j}\right)
$$

where $\pi_{j}$ is the prior probability that $x_{i}$ belongs to class $j, p\left(x_{i} \mid \tau_{j}\right)$ is chosen as Rayleigh distribution corresponding to the class $j$ is given by:

$$
p\left(x_{i} \mid \tau_{j}\right)=\frac{x_{i}}{\tau_{j}^{2}} e^{-x_{i}^{2} / 2 \tau_{j}^{2}}
$$

where $\tau_{j}$ denotes the covariance of the Rayleigh probability distribution function (PDF). However, Rayleigh distribution $p\left(x_{i} \mid \tau_{j}\right)$ is unbounded with support range $[0,+\infty)$. In the area of US images, each tissue is with different bounded support. To deal with this issue, BRMM is proposed thereof.

Let $\partial_{\Omega j}$ be the bounded support region, $\Omega_{j}$ denotes the $j$ th class corresponding to the region in image. The indicator function is introduced as follows:

$$
H\left(x_{i} \mid \Omega_{j}\right)= \begin{cases}1 & x_{i} \in \partial_{\Omega_{j}} \\ 0 & \text { otherwise }\end{cases}
$$

With the indicator function $H\left(x_{i} \mid \Omega_{j}\right)$ in (3) and the distribution $p\left(x_{i} \mid \tau_{j}\right)$ in (2), a bounded Rayleigh distribution $\Phi\left(x_{i} \mid \tau_{j k}\right)$ is given by:

$$
\Phi\left(x_{i} \mid \tau_{j k}\right)=\frac{p\left(x_{i} \mid \tau_{j k}\right) H\left(x_{i} \mid \Omega_{j}\right)}{\int_{\partial_{\Omega_{j}}} p\left(x \mid \tau_{j k}\right) d x}
$$

where $\int_{\partial_{\Omega_{j}}} p\left(x \mid \tau_{j k}\right) d x$ is the normalization constant, and it identifies the share of $p\left(x_{i} \mid \tau_{j k}\right)$ that belongs to the support region $\partial_{\Omega j}$. The idea to define $\Phi\left(x_{i} \mid \tau_{j k}\right)$ in (4) is because that each tissue in US images has bounded support. $\Phi\left(x_{i} \mid \tau_{j k}\right)$ is equal to $p\left(x_{i} \mid \tau_{j k}\right)$ inside the support region; and is zero, outside.

Next, in order to fit different shapes of US data such as non-symmetric and bounded support data, the proposed distribution $p\left(x_{i} \mid \Omega_{j}\right)$ is defined as:

$$
p\left(x_{i} \mid \Omega_{j}\right)=\sum_{k=1}^{K_{j}} \eta_{j k} \Phi\left(x_{i} \mid \tau_{j k}\right)
$$

where $K_{j}$ is the number of the bounded Rayleigh distributions that are used to model the label $\Omega_{j} . \eta_{j k}$ is the weighting factor that satisfies the following constraints:

$$
\eta_{j k} \geq 0 \text { and } \sum_{k=1}^{K_{j}} \eta_{j k}=1
$$

It is worth mentioning that $p\left(x_{i} \mid \Omega_{j}\right)$ always satisfies the condition of the probability density [1]:

$$
p\left(x_{i} \mid \Omega_{j}\right) \geq 0, \text { and } \int_{-\infty}^{+\infty} p\left(x_{i} \mid \Omega_{j}\right) d x=1
$$

We need parameters set $\Theta=\left\{\pi_{j}, \eta_{j k}, \tau_{j k}\right\}$ in order to maximize the likelihood function. The method will be elaborate in this section. The complete - data log likelihood can be written as:

$$
L(\Theta)=\sum_{i=1}^{N} \log \left[\sum_{j=1}^{L} \pi_{j}\left(\sum_{k=1}^{K_{i}} \eta_{j k} \frac{\Psi\left(x_{i} \mid \tau_{j k}\right) H\left(x_{i} \mid \Omega_{j}\right)}{\int_{\partial_{\Omega_{j}}} \Psi\left(x_{i} \mid \tau_{j k}\right) d x}\right)\right]
$$

Since the logarithm is a monotonically increasing function, it is more convenient to consider the negative logarithm 
of the likelihood function [9], as an error function. Maximizing the likelihood $L(\Theta)$ in (8) is equivalent to minimizing $J(\Theta)$ in $(9)$.

$$
J(\Theta)=-L(\Theta)=-\sum_{i=1}^{N} \log \left[\sum_{j=1}^{L} \pi_{j}\left(\sum_{k=1}^{K_{i}} \eta_{j k} \frac{\Psi\left(x_{i} \mid \tau_{j k}\right) H\left(x_{i} \mid \Omega_{j}\right)}{\int_{\partial_{\Omega_{j}}} \Psi\left(x_{i} \mid \tau_{j k}\right) d x}\right)\right]
$$

In order to minimize $J(\Theta)$, we define two variables $z_{i j}{ }^{(t)}$ and $y_{i j k}{ }^{(t)}$. The variables $z_{i j}{ }^{(t)}$ and $y_{i j k}{ }^{(t)}$ are defined as:

$$
z_{i j}^{(t)}=\frac{\pi_{j} \sum_{k=1}^{K_{j}} \eta_{j k} \Phi\left(x_{i} \mid \tau_{j k}\right)}{\sum_{m=1}^{L} \pi_{m}\left[\sum_{k=1}^{K_{j}} \eta_{m k} \Phi\left(x_{i} \mid \tau_{m k}\right)\right]}, y_{i j k}^{(t)}=\frac{\eta_{j k} \Phi\left(x_{i} \mid \tau_{j k}\right)}{\sum_{m=1}^{K_{j}} \eta_{j m} \Phi\left(x_{i} \mid \tau_{j m}\right)}
$$

Since $z_{i j}^{(t)}$ always satisfy the conditions $\sum_{j=1}^{L} z_{i j}^{(t)}=1$, Jensen's inequality in the form log is applied so that $\log \left(\sum_{j=1}^{L} z_{i j}^{(t)} s\right) \geq \sum_{j=1}^{L} z_{i j}^{(t)} \log (s)$, and also $y_{i j k}^{(t)}$ always satisfy $\sum_{k=1}^{K_{j}} y_{i j k}^{(t)}=1$ so that $\log \left(\sum_{k=1}^{K_{j}} y_{i j k}^{(t)} s\right) \geq \sum_{k=1}^{K_{j}} y_{i j k}^{(t)} \log (s)$. We can obtain:

$$
J(\Theta) \leq-\sum_{i=1}^{N} \sum_{j=1}^{L} z_{i j}^{(t)}\left\{\log \pi_{j}+\sum_{k=1}^{K_{i}} y_{i j k}^{(t)}\left[\log \eta_{j k}+\log \Phi\left(x_{i} \mid \tau_{j k}\right)\right]\right\}
$$

Minimizing $J(\Theta)$ in Eq. (9) is equivalent to minimizing the error function $E(\Theta)$ :

$$
\begin{aligned}
E(\Theta)= & -\sum_{i=1}^{N} \sum_{j=1}^{L} z_{i j}^{(t)}\left\{\log \pi_{j}+\sum_{k=1}^{K_{i}} y_{i k k}^{(t)}\left[\log \eta_{j k}+\log H\left(x_{i} \mid \Omega_{j}\right)\right.\right. \\
& \left.\left.+\log \Psi\left(x_{i} \mid \tau_{j k}\right)-\log \int_{\partial_{\Omega_{j}}} \Psi\left(x \mid \tau_{j k}\right) d x\right]\right\}
\end{aligned}
$$

So that, the parameters set $\Theta=\left\{\pi_{j}, \eta_{j k}, \tau_{j k}\right\}$ estimated by minimize $E(\Theta)$ in Eq. (12). At the $(t+1)$ iteration step, we consider the derivation of $E(\Theta)$ with $\tau_{j k}$ at first. Then, update $\pi_{j}$ and $\eta_{j k}$.

$$
\begin{gathered}
\tau_{j k}^{(t+1)}=\frac{\sum_{i=1}^{N} z_{i j}^{(t)} y_{i j k}{ }^{(t)}\left(x_{i}^{2} / 2\right)}{\sum_{i=1}^{N} z_{i j}{ }^{(t)} y_{i j k}{ }^{(t)}} \\
\pi_{j}^{(t+1)}=\frac{1}{N} \sum_{i=1}^{N} z_{i j}^{(t)}, \eta_{j k}^{(t+1)}=\frac{\sum_{i=1}^{N} z_{i j}^{(t)} y_{i j k}^{(t)}}{\sum_{i=1}^{N} z_{i j}^{(t)} \sum_{m=1}^{K_{j}} y_{i j m}{ }^{(t)}}
\end{gathered}
$$

\section{EXPERIMENTS}

In this section, we experimentally BRMM in a set of images. We also evaluate GMM, BGMM and RMM for comparison. The experiments have been developed in MATLAB R2013a, and are executed on an Intel i5 Core $2.8 \mathrm{GHz}$ CPU with 12.0GB RAM.

Firstly, we evaluate the performance of GMM, BGMM, RMM, and BRMM for synthetic US images that simulated from CT images [10]. For this experiment, we set $K_{j}=2$, and $L=2$. The segmentation results shown in Fig. 1. The first column shows original images and the second column shows ground truth. To demonstrate these segment results, red color denotes the target class and green color denotes the background. The third column denotes GMM with unbounded range $\partial_{\Omega_{j}} \in(-\infty,+\infty)$ while the fourth column denotes RMM with support range $\partial_{\Omega_{j}} \in[0,+\infty)$. The fifth column denotes BGMM with support range $\partial_{\Omega_{j}} \in[0,120]$ while the last column denotes BRMM is with bounded range $\partial_{\Omega_{j}} \in[0,120]$. As shown in Fig. 1, a visual inspection of the result of BRMM indicates that it yields better results than other methods. 


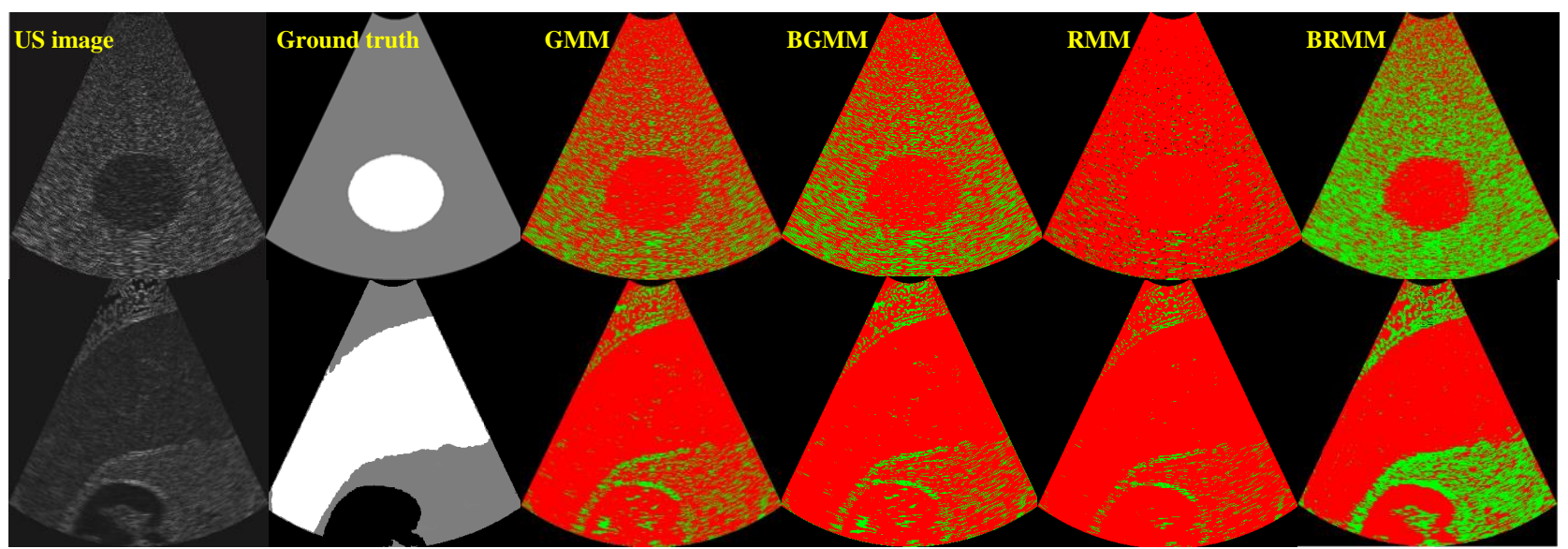

Figure 1. Mixture models for segmentation. (From left to right: original images, ground truth, GMM, BGMM, RMM and BRMM. Ground truth: white denotes target; gray denotes background. Segmentation: red denotes target; green denotes background. )

Table 1. Dice scores (DSC) indicating the segmentation accuracy using several mixture models

\begin{tabular}{c|c|c}
\hline Model/DSC & Elliptical Image & Abdomen Image \\
\hline GMM & 0.3551 & 0.7405 \\
BGMM & 0.3782 & 0.7986 \\
RMM & 0.3813 & 0.7930 \\
BRMM & 0.4264 & 0.8670 \\
\hline
\end{tabular}

We also evaluate the accuracy and computation efficiency of different models. Table 1 presents the Dice score (DSC) [11] between the segmentation results and the ground truth obtained on the simulated images. DSC takes values between 0 and 1, with values closer to 0 indicating an inferior segmentation result and values closer to 1 indicating better result. We can observe that BRMM yields the best segmentation results with the highest DSC values. For the elliptical image, the difference between GMM, BGMM, RMM and BRMM in terms of DSC value is $20.1 \%, 12.7 \%$ and $11.8 \%$, respectively. For the abdomen image, the difference is $17.1 \%, 8.5 \%$ and $9.3 \%$, respectively. We also evaluate the computation time for all methods. It is noted that BRMM is much faster than other methods. The computation time of BRMM is $1.516 \mathrm{~s}$. GMM is the slowest method that takes 301.58s while BGMM takes 180.65s. RMM takes 6.947s.

Then, we evaluate our model on real US images obtained by High-Intensity Focused Ultrasound (HIFU). HIFU ablation is a safe, feasible and cost-effective alternative for the treatment of many diseases. Since HIFU is a noninvasive therapeutic procedure, it applied for women patients with symptomatic uterine fibroids. Before the treatment, the focal areas of diseases could be accurately located in order to decrease the complexity during the operation. Fig. 2 presents the segmentation results. The first row of Fig. 2 shows uterine HIFU images. The boundary of uterine is blur and the region of uterine is inhomogeneous in original images. The second row shows segmentation results achieved by BRMM. These images show clearer boundary and homogeneous region of uterine. Homogeneous region of focal improves the treatment accuracy of HIFU. The computation time of BRMM are $1.0 \mathrm{~s}, 0.7 \mathrm{~s}, 0.8 \mathrm{~s}$, respectively, which make it more attractive for clinical application.

\section{CONCLUSION}

In this paper, we proposed a new finite bounded Rayleigh mixture model (BRMM) for medical US images segmentation. Rayleigh distribution is non-symmetric which has ability to fit the shape of medical ultrasound data. Each component of the proposed model is suitable for the ultrasound image segmentation. The BRMM also can improve accuracy and reduce computation time so that it is attractive to clinical application. Numerical experiments showed that the proposed model outperforms the state-of-art models in terms of time consumption and accuracy. 

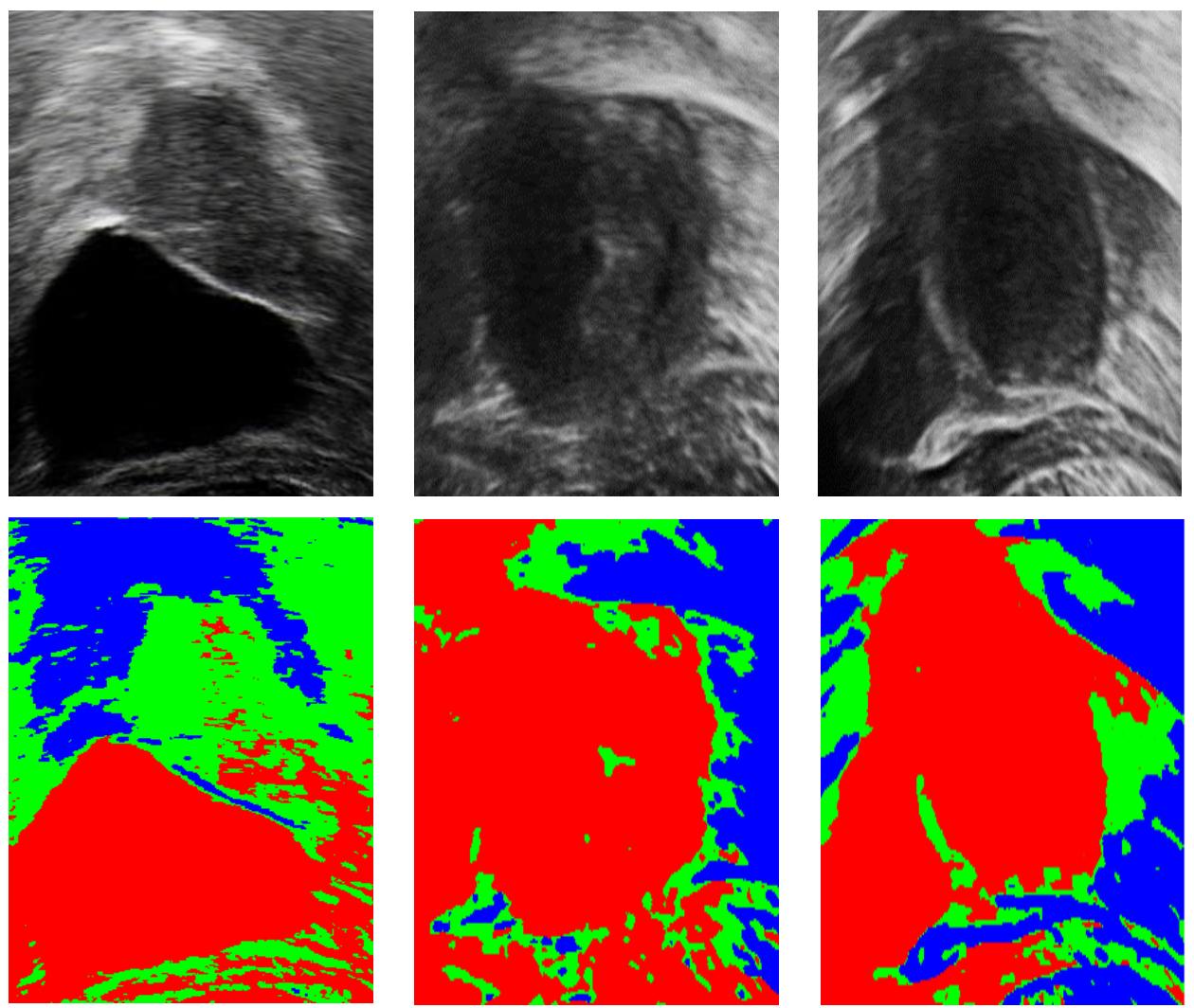

Figure 2. Results on uterine HIFU images. (The first row shows uterine US images; the second row shows segmentation results based on BRMM: red denotes uterine.).

\section{REFERENCES}

[1] M. Sonka, V. Hlavac, R. Boyle, Image processing, analysis, and machine vision, Cengage Learning, 2014.

[2] C. M. Bishop, Pattern Recognition and Machine Learning, Springer, 2006.

[3] A. P. Dempster, N. M. Laird, D. B. Rubin, "Maximum likelihood from incomplete data via the EM algorithm," J. Roy. Stat. Soc. B., vol. 39, pp. 1-38, 1977.

[4] R. F. Wagner, S. W. Smith, J. M. Sandrik, et al, "Statistics of speckle in ultrasound B-scans," IEEE Trans. Sonics Ultrason., vol. 30, no. 3, pp. 156-163, 1983.

[5] V. Damerjian, O. Tankyevych, N. Souag, et al, "Speckle characterization methods in ultrasound images-A review," IRBM, vol. 35, no. 4, pp. 202-213, 2014.

[6] V. Dutt, J. F. Greenleaf, "Ultrasound echo envelope analysis using a homodyned K distribution signal model," Ultrasonic Imaging, vol. 16, no. 4, pp. 265-287, 1994.

[7] P. M. Shankar, "Ultrasonic tissue characterization using a generalized Nakagami model," IEEE Trans. Ultrason., Ferroelet., Freq. Control, vol. 48, no. 6, pp. 1716-1720, 2001.

[8] J. C. Seabra, F. Ciompi, O. Pujol, et al, "Rayleigh mixture model for plaque characterization in intravascular ultrasound," IEEE Trans. Biomed. Eng., vol. 58, no. 5, pp. 1314-1324, 2011.

[9] T. M. Nguyen, Q. M. J. Wu, "Bounded Asymmetrical Student's-t Mixture Model,” IEEE Trans. Cybern., vol. 44, no. 6, pp. 857-869, 2014.

[10] J. L. Dillenseger, S. Laguitton, E. Delabrousse, "Fast simulation of ultrasound images from a CT volume," Comput. Biol. Med., vol. 39, no. 2, pp. 180-186, 2009.

[11]L. R. Dice, "Measures of the Amount of Ecologic Association between Species," Ecology, vol. 26 no. 3, pp. 297 $302,1945$. 